\title{
Dietary fat and obesity: still an important issue
}

\author{
Arne Astrup \\ Department of Human Nutrition and Centre for Advanced Food Studies, The Royal Veterinary and Agricultural \\ University, Frederiksberg, Denmark
}

\section{Abstract}

The role of a reduction in dietary fat for weight loss and maintenance should be assessed by evidence-based principles giving most weight to randomized clinical trials. Four meta-analyses examined weight changes on ad libitum fat-reduced diets in intervention trials lasting for up to 1 year, and they all demonstrated a 3-4 kg larger weight loss on the fat-reduced than on the normal-fat diet in normal-weight and overweight subjects. The analyses also show a dose-response relationship, i.e. the reduction in percentage energy as fat is positively associated with weight loss. Weight loss is also positively related to initial weight: a $10 \%$ reduction in dietary fat is predicted to produce a $4-5 \mathrm{~kg}$ weight loss in an individual with a body mass index of $30 \mathrm{~kg}$ $\mathrm{m}^{-2}$. The outcome of the meta-analysis of trials with long-term follow-up included only six studies, and none of the trials had an active intervention throughout the period. Short-term trials clearly demonstrate that the non-fat diet components are at least as important for body-weight regulation as the fat content. Sugar in beverages is less satiating and more obesity promoting than sugar in solid foods, and replacement of energy from fat by sugar derived from sweetened beverages is not likely to produce weight loss. Protein is more satiating and thermogenic than carbohydrates, and a fat-reduced diet with a high protein content (20-25\% of energy) may increase the efficacy of fat-reduced diets markedly. Whereas the glycaemic index of the carbohydrate may play a role for cardiovascular risk factors, there is very little evidence to support that low glycaemic index foods facilitate weight control. The evidence linking particular fatty acids to body fatness is weak. If anything, monounsaturated fatty acids (MUFA) may be more fattening than polyunsaturated and saturated fats, and no ad libitum dietary intervention study has shown that a normal-fat, high-MUFA diet is equivalent or superior to a low-fat diet in the prevention of weight gain and obesity. The current evidence strongly supports a diet with reduced content of fat and sugar-rich beverages, and more carbohydrates, rich in fibre and grain (whole-grain foods, fruit and vegetables) and protein (lean meat and dairy products) as the best choice for the prevention of weight gain, obesity, type 2 diabetes and cardiovascular disease. The use of a normal-fat, high-MUFA diet needs more evidence from randomized ad libitum dietary intervention trials before it can be recommended to the public.

Keywords: fat-reduced diets; food pyramid; glycaemic index; low-fat diets; protein; weight loss

\section{The currently recommended diet}

To prevent cardiovascular disease, cancer, obesity and type 2 diabetes, it is recommended that dietary fat should be reduced from the current $35-45 \%$ of the total energy content in most Western diets to below $30 \%(1,2)$. However, observational epidemiology does not support that this reduction in total fat content is a prerequisite for primary and secondary prevention of cardiovascular disease, because monounsaturated fatty acids (MUFA) and polyunsaturated fatty acids (PUFA) are less atherogenic than saturated fat and even carbohydrate (3). This has been translated into the public health recommendations by Willett and co-workers that the total fat content of the diet is unimportant, and that trans fat and saturated fat just need to be replaced by MUFA and PUFA. They conclude that recommending a reduction in total dietary fat is misleading and unnecessary, and they point to starchy carbohydrates with a high glycaemic index (GI) as posing a greater risk of producing weight gain and obesity, and increasing cardiovascular risk (4).

The present review examined the evidence in favour of a fat-reduced diet $(25-30 \%$ energy from fat) versus a normal-fat (30-40\%), high-MUFA diet for the prevention of weight gain and obesity, type 2 diabetes and to some extent cardiovascular disease.

The evidence-based principle was used to assess the strength of the existing documentation to 
substantiate whether one of the diets is superior to the other (5). Notably, meta-analysis of randomized controlled trials is recognized as being the strongest evidence, followed by experimental studies, and finally by observational epidemiological studies and personal opinions.

\section{How does dietary fat influence energy balance?}

A link between dietary fat content and obesity must be exerted through an effect of the ingested fat on energy balance, and a positive energy balance can be promoted by overconsumption of energy, e.g. owing to a lower satiating effect per joule of a highfat versus a low-fat diet. Moreover, energy from fat may be more effectively absorbed from the intestine than carbohydrate and protein, and fat may also reduce energy expenditure, e.g. by a lower thermogenic effect of fat compared with carbohydrate and protein. All the evidence points to fat exerting its effect on energy balance by affecting spontaneous energy intake, and the relation between dietary fat and body fat should therefore be studied under $a d$ libitum conditions, where the studied individuals have free access to food.

There is a robust series of studies showing that most of the fattening effect of dietary fat is linked to the higher energy density of fatty foods than carbohydrate- and protein-rich foods (for review see Ref. (6)).

\section{The four meta-analyses of randomized clinical trials on fat-reduced versus normal-fat diets}

The author is aware of four meta-analyses of controlled trials comparing low-fat diets with normal-fat diets as a control under ad libitum conditions. In a systematic review and meta-analysis based on 28 intervention trials, Bray and Popkin found that a reduction of $10 \%$ in the proportion of energy from fat was associated with a reduction in weight of $16 \mathrm{~g} \mathrm{day}^{-1}$. This corresponds to a weight loss of $2.9 \mathrm{~kg}$ over 6 months (7). The first metaanalysis by the present author included a total of 1728 individuals, and 13 studies were randomized controlled trials. The control groups were advised either to continue their regular diet or to consume a diet with a fat content comparable to that of the background population. The low-fat interventions produced a weight loss of $2.4 \mathrm{~kg}$ more than in the control groups [95\% confidence interval $(95 \% \mathrm{CI})$ $1.9-2.9, p<0.0001]$ in the fixed effects analysis, and
$2.5 \mathrm{~kg}$ more $(95 \%$ CI $1.5-3.5, p<0.0001)$ in the random effects analysis. In a simple correlation analysis the major determinant of the weight loss difference was pretreatment body weight $(r=0.52$, $p<0.05)$. There was a dose-response relationship between the reduction in percentage dietary fat intake and weight loss after adjustment for pretreatment body weight $(r=0.66, p<0.005)$. With no change in percentage dietary fat intake, no weight change occurred (8).

The third meta-analysis included 37 dietary intervention studies. Weight loss in the intervention groups was $2.8 \mathrm{~kg}$ larger than in the control groups, and there was a highly significant relation between reduction in dietary fat and weight loss (9). For every $1 \%$ decrease in energy from fat there was a $0.28 \mathrm{~kg}$ decrease in body weight.

The fourth meta-analysis was by the author's group, and included new trials and excluded any trial in which the intervention group was instructed to increase physical activity. Sixteen trials (duration of $2 \pm 12$ months) with 19 intervention groups, were included, giving a total of 1910 individuals participating in studies. Weight loss was not the primary aim in 11 studies. Before the interventions the mean proportion of dietary energy from fat in the studies was $37.7 \%$ in the low-fat groups and $37.4 \%$ in the control groups. The low-fat intervention produced a mean fat reduction of $10.2 \%(8.1-12.3)$. Low-fat intervention groups showed a greater weight loss than control groups $(3.2 \mathrm{~kg}, 1.9-4.5 \mathrm{~kg} ; p<$ 0.0001 ), and a greater reduction in energy intake (1138 $\mathrm{kJ}$ day $^{-1}, 564-1712 \mathrm{~kJ}$ day $\left.^{-1} ; p<0.002\right)$. A pretreatment body weight $10 \mathrm{~kg}$ higher than the average was associated with a $2.6 \mathrm{~kg}$ greater difference in weight loss (10). These four metaanalyses thus consistently show that a reduction in dietary fat without restriction of total energy intake causes a reduction in caloric intake and weight loss in a dose-dependent fashion and may produce a modest, but clinically relevant, weight loss in overweight subjects.

The fifth meta-analysis: does fat reduction work in the long term?

Pirozzo et al. (11) conducted a meta-analysis to determine the effectiveness of fat-reduced diets in achieving sustained weight loss when used for the expressed purpose of weight loss in obese or overweight subjects. The analysis included six trials, and 
they concluded that low-fat diets are as efficacious as other weight-reducing diets for achieving sustained weight loss, but not more so. Hill and Astrup (12) expressed some concerns about the conclusions drawn from the meta-analysis by Pirozzo et al. (11). The major problem is the very few trials that met the inclusion criteria set by the authors. Several trials supportive of low-fat diets in weight management did not meet these criteria. This suggests that despite the tremendous interest and debate about the best diets to recommend for long-term weight loss, there are surprisingly few studies that address this issue. Secondly, there are some important differences in study design among the six studies. For example, two studies were of only 3-4 months' duration, three trials lasted for only 6 months, and one was of 18 months' duration. It is very difficult to compare a study where intervention was 3 months with one where intervention was 18 months. While the authors aimed to assess the effectiveness of the different diets on sustained weight loss, none of the studies had active intervention during the follow-up period. Thus, the questions addressed is not whether low-fat diets can sustain weight loss but whether a few months of low-fat eating can lead to sustained weight loss. To answer the first question, studies would have to continue active intervention (fat-reduced versus normal-fat) for sustained periods. This was not done in any of the six studies included in this meta-analysis, but can be found in other trials $(13,14)$.

\section{Does 3-5 kg weight loss really matter?}

A sustained weight loss of $3-5 \mathrm{~kg}$ in obese at high risk for diabetes or cardiovascular disease is sufficient to reduce the incidence of type 2 diabetes by $40-60 \%(15-17)$ and reduce the incidence of cardiac events by around $40 \%(13,14,18)$, and increasing physical activity amplifies the effect (9). Type 2 diabetes is associated with an almost 10 year reduction in life expectancy, mainly due to increased cardiovascular mortality, and its prevention therefore has substantial health benefits.

In addition, typical weight loss of $3-5 \mathrm{~kg}$ produced by ad libitum low-fat diets is more likely to be an underestimation of the true effect. Adherence to low-fat diets can be difficult in a "toxic high-fat, soft-drink environment". There is good evidence to demonstrate the necessity of some sustained intervention therapy in order to counteract the tendency to fall back into usual dietary habits. Consequently, compliance and diet adherence become key issues to consider when assessing the efficacy of fat-reduced diets in long-term weight management.

Several trials have provided some evidence to support the theory that incomplete dietary compliance tends to underestimate the real effect of fat reduction. Swinburn et al. found that weight loss on an ad libitum low-fat diet was $3.3 \mathrm{~kg}$ after 1 year (19). However, if the subjects in the intervention group are stratified according to compliance assessed by attendance at the monthly meetings and completion of the diet diaries, one gains a better idea about the efficacy of the low-fat diet in producing weight loss (Fig. 1). While those in the less compliant group lost only about $1 \mathrm{~kg}$, the more compliant group lost almost $6 \mathrm{~kg}$ after 1 year (19). Moreover, it is evident that many people think that they are consuming a low-fat diet but are not aware of many of the foods they eat are high in fat. Such factors will contribute to underestimate the real efficacy of low-fat diets. The follow-up in this trial clearly shows that unless the intervention is reinforced regularly the patients will inevitably relapse (Fig. 1). In this aspect, dietary treatment does not differ from other medical treatment: it only works for as long as it is taken!

\section{Change in Weight}

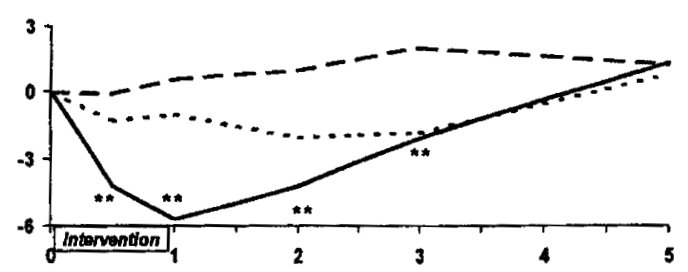

Fig. 1. Changes in body weight in a control group consuming a normal-fat diet ad libitum (---), and in the least (...) and most (-) compliant individuals in the ad libitum low-fat intervention groups. The results indicate that the true efficacy of the low-fat diet is not the $3.3 \mathrm{~kg}$ weight loss observed after 1 year in the whole intervention group, but rather the $6 \mathrm{~kg}$ weight loss seen in the most compliant group. It is also obvious from the weight course that the effect of the diet change is effective only as long as the intervention is exerted. As soon as the intervention was stopped weight regain occurred, and after 5 years no effect remained compared with the control group. As overweight is a chronic condition and a permanent lifestyle change is needed, future interventions should include reinforcement sessions to prevent relapse. ${ }^{* *}$ indicates difference between intervention and controls group at the $\mathrm{P}<0.01$ level. [Reproduced with permission from Swinburn et al. (19)]. 


\section{The role of different carbohydrates: a role for fibre and whole-grain foods}

\section{Glyacemic index of foods}

In Willetts' suggestion for a new "inversed" food pyramid, it is stressed that the carbohydrate-rich foods should predominantly be whole-grain and fibre rich, with a low GI (4). The long-term effects of a diet containing low or high GI foods on body weight have been very poorly investigated $(20,21)$, and the interpretation of the current body of evidence is conflicting (22). In the majority of the published studies a low GI diet has been found to produce favourable effects on risk factors of diabetes and cardiovascular diseases compared with a high GI diet. Since most of the intervention studies have used diets aiming at energy balance or energy reduction the effects on body weight are, however, still unclear, and recent evidence suggests that the GI of composite meals is unpredictable based on the tables providing GI values for individual foods (23).

\section{Complex versus simple carbohydrates}

The role of different types of carbohydrate in the regulation of body weight has not been investigated as thoroughly as the role of the total dietary fat content. A few recent studies have illuminated this area with regard to carbohydrate structure (e.g. simple/complex carbohydrates, sucrose/starch). The largest and most recent is the CARMEN multicentre trial, which involved a total of 316 overweight subjects in five different countries (24). In this study, the impact of 6 months' ad libitum intake of low-fat diets, rich in either simple or complex carbohydrates, on energy intake, body weight and blood lipids was investigated. The results showed that both low-fat diets reduced body weight by 1.6 $\mathrm{kg}$ and $2.4 \mathrm{~kg}$, respectively, compared with the normal-fat control diet. The slightly lower weight loss on simple carbohydrates than on complex carbohydrates did not reach statistical significance. Furthermore, no detrimental effects on blood lipids were observed during either of the two carbohydrate diets. According to this study, the type of carbohydrate (simple sugars versus complex carbohydrates) does not seem to have much importance for bodyweight regulation and risk factors, as previously suggested.

As alluded to above, the low energy density of carbohydrates compared with fat is important. One gram of carbohydrate $\left(17 \mathrm{~kJ} \mathrm{~g}^{-1}\right)$ provides less than half the calories of $1 \mathrm{~g}$ of fat $\left(37 \mathrm{~kJ} \mathrm{~g}^{-1}\right)$. This means that few calories are consumed in a carbohydrate-rich meal than in a fat-rich meal of similar weight. A starch- and fibre-rich diet is often comprised of a surprisingly large volume of foods. In one long-term study using such a diet, the volunteers had problems consuming the large volume of food, especially on the diets composed for the volunteers with the highest energy requirements.

\section{A role for whole grain and fibre?}

A decreased energy availability of a high-fibre diet may also play a role. The ingestion of $64 \mathrm{~g}$ compared with $34 \mathrm{~g}$ dietary fibre day $^{-1}$ for 10 weeks in lean healthy males resulted in a 5.7\% lower protein utilization and in a 3.0\% lower fat utilization (25). Total energy utilization was $2.9 \%$ lower, equal to $400 \mathrm{~kJ} \mathrm{day}^{-1}$ (25). A theoretical calculation showed that if the fibre intake in a typical American diet was increased from $18 \mathrm{~g} \mathrm{day}^{-1}$ to 36 $\mathrm{g} \mathrm{day}^{-1}$ the amount of metabolizable energy would be reduced by $540 \mathrm{~kJ}^{\text {day }}{ }^{-1}$ (26).

A long-term, randomized dietary intervention trial in patients with cardiovascular disease showed that a modest $6 \%$ point reduction in dietary fat, combined with increases in fish, fruit, vegetables, pulses and nuts, is associated with a weight loss of 4 $\mathrm{kg}$ over 1 year and a reduction in cardiac events and mortality by around $40 \%$ (13). In a recent trial that enrolled 1000 patients the control group was instructed to follow the National Cholesterol Education Program Step 1 Prudent Diet, which recommends $<30 \%$ of energy from fat and $<10 \%$ from saturated fat (14). The intervention group was instructed to follow the same diet and in addition to consume at least $250-300 \mathrm{~g}$ of fruit, $125-150 \mathrm{~g}$ of vegetables, 25-30 g of walnuts or almonds, and $400-500 \mathrm{~g}$ of whole grains, legumes, rice, maize or wheat. Together, this achieved a 3\% point reduction in total fat content, together with a significant increase in $\alpha$-linolenic acid. The cardioprotective effect previously reported was confirmed, and the intervention group spontaneously reduced their energy intake, resulting in a weight loss of $3 \mathrm{~kg}$ over 2 years. These trials suggest that future dietary intervention trials should focus more on a combination of fat reduction and increased intake of wholegrain and fibre-rich foods. 


\section{Sucrose versus artificial sweeteners}

It has been speculated that the removal of sucrose (carbohydrate) from the diet will increase the relative dietary fat content, which would then result in increased energy intake and body weight in the long term. The number of long-term intervention studies without caloric restriction is small, and none has lasted for more than 3 weeks $(27,28)$. These intervention studies suggest that an increased intake of artificial sweeteners decreases energy intake and body weight compared with sucrose. The author's group investigated the effect of 10 week supplementation with either sucrose or artificial sweeteners on ad libitum food intake and body weight in two groups of overweight subjects. About $80 \%$ of the supplements were given as drinks and $28 \%$ of the caloric intake came from sucrose in the sucrose group. Similar food items and volumes were given to the two groups. An increase in total energy intake $\left(2.6 \mathrm{MJ}\right.$ day $\left.^{-1}\right)$, body weight $(1.6 \mathrm{~kg})$ and fat mass $(1.3 \mathrm{~kg})$ was seen in the sucrose group after 10 weeks, whereas a decrease in body weight and fat mass $(1.0 \mathrm{~kg}$ and $0.3 \mathrm{~kg})$ was seen in the artificial sweetener group (21). One likely reason for the increased energy intake and body weight in the sucrose group is that about $70 \%$ of the sucrose came from fluids. Calories from fluids have been shown to be less satisfying than solid foods, and it is easier to overconsume energy from drinks than from solids (29). That sucrose from fluids may be fattening was also suggested by a recent observational, prospective study in children, reporting that those with a high intake of sugar-sweetened drinks were at an increased risk of becoming overweight (30). It may therefore be advisable for overweight subjects to choose drinks and foods containing artificial sweeteners rather than sucrose in order to prevent weight gain.

\section{Protein}

There is some concern that a high protein intake in infant formulae and during growth may increase the susceptibility to weight gain and obesity. However, a new study suggests that a high protein intake may be associated with a higher body mass index (BMI) owing to a positive relationship with the size of the fat-free mass, not with the amount of body fat (31). There is also a large body of experimental data to suggest that protein has a higher satiating power per calorie than carbohydrate and fat in adults. The impact on obesity and risk factors of replacing carbohydrate with protein in ad libitum low-fat diets has been addressed in only one clinical trial. Two fat-reduced diets ( $30 \%$ of total energy), a highcarbohydrate diet (protein 12\% of total energy) and a high-protein diet (protein $25 \%$ of total energy) were compared in 65 obese patients (32). Weight loss after 6 months was $5.1 \mathrm{~kg}$ in the highcarbohydrate and $8.9 \mathrm{~kg}$ in the high-protein group, and more subjects lost $>10 \mathrm{~kg}$ in the high-protein group $(35 \%)$ than in the high-carbohydrate group $(9 \%)$. The protein-rich diet had no adverse effect on blood lipids, homocysteine levels (33), renal function (34) or bone mineral density (35). Replacement of some dietary carbohydrate by protein in ad libitum, low-fat diets may improve weight loss. More freedom to choose between protein-rich and complex carbohydrate-rich foods may encourage obese subjects to choose more lean meat and dairy products, and hence improve adherence to low-fat diets in weight-reduction programmes.

\section{Effects of different types of fat on energy balance}

Although the same quantities of different types of fat contain almost the same amounts of energy, differences may exist in their potential to influence energy balance. Thus, energy expenditure as well as satiety, both of which influence the energy balance of ad libitum low-fat diets and weight-maintenance diets, have shown to be affected by the quality of fat.

It is known from animal studies that rats fed a diet rich in safflower oil (PUFA) demonstrate less accumulation of body fat than rats fed a diet rich in beef tallow (saturated fat) $(36,37)$. This is probably due to a higher diet-induced thermogenesis, an elevated fat oxidation and a higher sympathetic activity $(36,38)$. MUFA also seems to increase body weight more than PUFA (37). Other studies also report differences in fat accumulation, body-fat distribution and oxidation rates as a result of diets varying in fatty acid composition, chain length and saturation (39).

In humans, in a cross-sectional, observational study, strong positive associations between the intake of monounsaturated fats (MUFA) and different indices of adiposity were found, whereas high intakes of polyunsaturated fats (PUFA) and saturated fat were only weakly related to adiposity (40). Similarly, in a cohort of women, all lipids other than vegetable fat were positively related to BMI. Furthermore, the strongest positive association 
between a nutrient and BMI was found to be the intake of trans fatty acids (41). A study from Willett's group also suggests that MUFA may be more fattening (42).

A study on the effect of high-fat meals, differing in fatty acid composition, on postingestive satiety in lean subjects found that MUFA induced a lower level of satiety and a larger subsequent energy intake than PUFA and saturated fat (43). Intestinal infusions of linoleic acids concordantly result in a lower subsequent food intake than oleic and stearic acids. However, the different effects on energy expenditure, appetite and ad libitum energy intake were not confirmed in a recent study performed with overweight subjects, suggesting that differences exists between subject groups (44).

Together, these preliminary reports suggest that some differences between fatty acids are apparent, but until clinical trials based on longer term interventions have been conducted some caution should be taken in recommending specific fat types in preference to others, e.g. replacement of PUFA with MUFA in diets for individuals susceptible to weight gain and obesity, despite the apparently more neutral effects of MUFA reported in some studies in relation to insulin resistance, type 2 diabetes, cardiac heart disease and cancer. The author is aware only of the trial reported by Wolever et al. (45), who randomized subjects to three different ad libitum diets, one normal-fat high MUFA diet and two different fat-reduced diets, with either high or low GI. After 16 weeks, body weight and insulin resistance were increased in the high-MUFA diet compared with the fat-reduced diets (46).

\section{Conclusions}

Ad libitum consumption of diets with a reduced fat content (25-30\% of energy), and high in protein and fibre-rich, whole-grain carbohydrates, contributes to the prevention of weight gain in normalweight subjects, and causes a spontaneous weight loss of 3-4 kg in overweight subjects (Table 1). The modest weight loss has proven sufficient to reduce the incidence of type 2 diabetes and cardiac events among subjects at high risk.

Because type 2 diabetes is associated with an increased cardiovascular mortality and a 10 year reduced longevity, the prevention of this disease will substantially justify the use of low-fat diets for the prevention of cardiovascular disease. A Mediterra- nean fat-reduced diet may leave room for plenty of fruits, vegetables and fish, and such a diet has been shown to reduce total mortality by $45-60 \%$ in individuals with ischaemic heart disease (2).

The inverted food pyramid proposed by Willett et al. has so far not been backed up by randomized clinical trials that support its efficacy for weight control. Whereas it is very likely that an increased consumption of whole-grain and fibre-rich foods may contribute to the prevention of weight gain and obesity, the recommendation of increased intake of fat (MUFA and PUFA) from plant oils, nuts, etc., should be viewed with caution as this clearly poses a risk of producing weight gain. Preliminary evidence supports that this might be the case (47) and that the weight gain is accompanied by the expected increased risk of complications.

In summary, more long-term, randomized clinical trials are desperately needed to study the impact of diet composition on weight loss, on maintenance of weight loss and on primary prevention of weight gain. Further, weight management is not simply a function of the type of diet eaten, but depends on the amount of physical activity performed. Most studies of diet composition have not included efforts to increase physical activity. Until more well-designed studies have examined the long-term impact of diet composition on body weight, one should not throw the baby (the fat-reduced diet) out with the bathwater. The author agrees with Willett that many of the previous randomized dietary intervention trials have many shortcomings, which should be taken into consideration in the design of future trials:

- lack of similar attention, food provision and number of visits in the intervention and control groups

- lack of compliance assessment by the use of biological markers

- lack of standardization of the non-fat dietary component

- lack of detailed description of other lifestyle changes in the intervention group

- need for more focus on gene-nutrient interaction?

\section{Acknowledgements}

Supported by grants from the Danish Medical Research 
Table I. Evidence for the importance of various dietary factors for body-weight regulation and obesity using evidence-based principles

\begin{tabular}{|c|c|c|c|}
\hline \multirow[t]{2}{*}{ Dietary factor } & \multicolumn{2}{|l|}{ Association with obesity } & \multirow[t]{2}{*}{ Level of evidence } \\
\hline & Short term ( $<6$ months) & Long term ( $>6$ months) & \\
\hline Fat (energy density) & +++ & + & $A(I A)$ \\
\hline Dietary fibre & - & & $\mathrm{A}(\mathrm{IB})$ \\
\hline Types of carbohydrate in solid foods/glycaemic index & 0 & 0 & $A(I B)$ \\
\hline Sugar-sweetened soft drink/fruit juices & ++ & $?$ & $A(I B)$ \\
\hline Artificial sweeteners & - & ? & $A(I B)$ \\
\hline Fruit and vegetables & $?$ & $?$ & $A(I B)$ \\
\hline Protein & - & $(-)$ & $A(I B)$ \\
\hline High MUFA diet (Willett diet) & + & $?$ & $A(I B)$ \\
\hline Palatability & $?$ & $?$ & - \\
\hline Calcium & - & & B (lla) \\
\hline Capsaicin, etc. & & & B (IIb) \\
\hline
\end{tabular}

Category A: evidence based on randomized clinical trials (IA: meta-analyses; IB: at least one trial); category B: evidence based on non-randomized trials and mechanistic studies. (IIA: evidence from at least one non-randomized controlled clinical trial; IIB: at least one other trial quasi-experimental design); MUFA: monounsaturated fatty acid; + : positive effect on body weight; -: negative effect on body weight (numbers of symbols indicate the strength of the effect).

Council and the Danish Food Technology and Nutrition Program (FØTEK).

\section{References}

1. National Institutes of Health. The practical guide: identification, evaluation, and treatment of overweight and obesity in adults. Publication No. 00-4084. NIH Bethesda, Maryland, USA; 2000.

2. Eurodiet core report. Nutrition and diet for healthy lifestyles in Europe: science and policy implications. Public Health Nutr 2001; 4: 265-73.

3. Willett WC. Is dietary fat a major determinant of body fat? Am J Clin Nutr 1998; 67: 556S-62S.

4. Willett WC, Stampfer MJ. Rebuilding the food pyramid. Sci Am 2002; 297: 64-71.

5. Eccles M, Freemantle N, Mason J. North of England evidence based guidelines development project: methods of developing for efficient drug use in primary care. $\mathrm{Br}$ Med J 1998; 316: 1232-5.

6. Rolls BJ, Hammer VA. Fat, carbohydrate, and the regulation of energy intake. Am J Clin Nutr 1995; 62(Suppl 5): 1086S-1095.

7. Bray GA, Popkin BM. Dietary fat intake does affect obesity. Am J Clin Nutr 1998; 68: 1157-73.

8. Astrup A, Ryan L, Grunwald GK, et al. The role of dietary fat in body fatness: evidence from a preliminary meta-analysis of ad libitum low fat dietary intervention studies. Br J Nutr 2000; 83(Suppl 1): S25-32.

9. Yu-Poth S, Zhao G, Etherton T, et al. Effects of the National Cholesterol Education Programs step I and step II dietary intervention programs on cardiovascular disease risk factors: a meta-analysis. Am J Clin Nutr 1999; 69: 632-46.

10. Astrup A, Grunwald GK, Melanson EL, et al. The role of low-fat diets in body weight control: a meta-analysis of ad libitum intervention studies. Int J Obes 2000; 24: $1545-52$.
11. Pirozzo S, Summerbell C, Cameron C, Galsziou P. Should we recommend low-fat diets for obesity? Obes Rev 2003; 4: 83-90.

12. Hill JO, Astrup A. What diets should we be recommending for obesity? Obes Rev 2003; 4: 77-8.

13. Singh RB, Rastogi SS, Verma R, et al. Randomised controlled trial of cardioprotective diet in patients with recent acute myocardial infarction: result of one-year follow up. Br Med J 1992; 304: 1015-9.

14. Singh RB, Dubnov G, Niaz MA, et al. Effect of an Indo-Mediterranean diet on progression of coronary artery disease in high risk patients (Indo-Mediterranean Diet Heart Study): a randomised single-blind trial. Lancet 2002; 360: 1455-61.

15. Tuomilehto J, Lindström J, Eriksson JG, et al. Prevention of type 2 diabetes mellitus by changes in lifestyle among subjects with impaired glucose tolerance. N Engl J Med 2001; 344: 1343-50.

16. Knowler WC, Barrett-Connor E, Fowler SE, et al. Reduction in the incidence of type 2 diabetes with lifestyle intervention or metformin. $\mathrm{N}$ Engl $\mathrm{J}$ Med 2002; 346: 393-403.

17. Sjöström L, Togerson JS, Hauptman J, Boldrin M. XENDOS - a landmark study: xenical in the prevention of diabetes in obese subjects. In: Abstracts from the Satellite Symposium New Frontiers in Weight Management, 9th International Congress on Obesity, São Paulo, Brazil, 2002.

18. Ornish D, Brown SE, Scherwitz LW, et al. Can lifestyle changes reverse coronary heart disease? Lancet 1990; 336: $129-33$.

19. Swinburn BA, Metcalf PA, Ley SJ. Long-term (5-year) effects of a reduced-fat diet intervention in individuals with glucose intolerance. Diabetes Care 2001; 24: 6196124.

20. Pi-Sunyer FX. Glycemic index and disease. Am J Clin Nutr 2002; 76: 290S-8S. 
21. Raben A, Vasilaras TH, Møller AC, Astrup A. Sucrose compared with artificial sweeteners: different effects on ad libitum food intake and body weight after $10 \mathrm{wk}$ of supplementation in overweight subjects. Am J Clin Nutr 2002; 76: 721-9.

22. Pawlak DB, Ebbeling CB, Ludwig DS. Should obese patients be counselled to follow a low-glycaemic index diet? Yes. Obes Rev 2002; 4: 235-44.

23. Møller BK, Flint A, Pedersen D, et al. Prediction of glycemic index. European Congress on Obesity. Int $\mathbf{J}$ Obes 2003; in press.

24. Saris WHM, Astrup A, Prentice AM, et al. Randomized controlled trial of changes in dietary carbohydrate/fat ratio and simple vs. complex carbohydrates on body weight and blood lipids: the CARMEN study. Int $\mathbf{J}$ Obes 2000; 24: 1310-8.

25. Miles C. The metabolizable energy of diets differing in dietary fat and fiber measured in humans. J Nutr 1992; 122: 306-11.

26. Baer DJ, Rumpler WV, Miles CW, Fahey GC Jr. Dietary fiber decreases the metabolizable energy content and nutrient digestibility of mixed diets fed to humans. J Nutr 1997; 127: 579-86.

27. Tordoff MG, Alleva M. Effect of drinking soda sweetened with aspartame or high-fructose corn syrup on food intake and body weight. Am J Clin Nutr 1990; 51: $963-9$.

28. Porikos KP, Hesser MF, van Itallie TB. Caloric regulation in normal-weight men maintained on a palatable diet of conventional foods. Physiol Behav 1982; 29: $293-$ 300 .

29. Di Meglio DP, Mattes RD. Liquid versus solid carbohydrate: effects on food intake and body weight. Int $\mathbf{J}$ Obes 2000; 24: 794-800.

30. Ludwig DS, Peterson KE, Gortmaker SL. Relation between consumption of sugar-sweetened drinks and childhood obesity: a prospective, observational analysis. Lancet 2001; 357: 505-8.

31. Hoppe C, Mølgaard C, Juul A, Michaelsen KF. Protein intake in infancy is related to body size but not to adiposity in 10-y old children. Int J Obes 2001; 25(Suppl 2): S64.

32. Skov AR, Toubro S, Rønn B, et al. Randomized trial on protein versus carbohydrate in ad libitum fat reduced diet for the treatment of obesity. Int J Obes 1999; 23: $528-36$

33. Haulrik N, Toubro S, Dyerberg J, et al. Effect of protein and methionine intakes on plasma homocysteine concentrations: a 6-mo randomized controlled trial in overweight subjects. Am J Clin Nutr 2002; 76: 1202-6.

34. Skov AR, Toubro S, Bülow J, et al. Changes in renal function during weight loss induced by high vs. lowprotein low-fat diets in overweight subjects. Int $\mathbf{J}$ Obes 1999; 23: 1170-7.
35. Skov AR, Haulrik N, Toubro S, et al. Effect of protein intake on bone mineralisation during weight loss: a 6month trial. Obes Res 2002; 10: 432-8.

36. Shimomura Y, Tamura T, Suzuki M. Less body fat accumulation in rats fed a safflower oil diet than in rats fed a beef tallow diet. J Nutr 1990; 120: 1291-6.

37. Dulloo AG, Mensi N, Seydoux J, Girardier L. Differential effects of high-fat diets varying in fatty acid composition on the efficiency of lean and fat tissue deposition during weight recovery after low food intake. Metabolism 1995; 44: 273-9.

38. Matsuo T, Shimomura Y, Saitoh S, et al. Sympathetic activity is lower in rats fed a beef tallow diet than rats fed a safflower oil diet. Metabolism 1995; 44: 9349.

39. Hill JO, Peters JC, Lin D, et al. Lipid accumulation and body fat distribution is influenced by type of dietary fat fed to rats. Int J Obes 1993; 17: 223-36.

40. Doucet NA, White MD, Després J-P, et al. Dietary fat composition and human adiposity. Eur $\mathbf{J}$ Clin Nutr 1998; 52: 2-6.

41. Colditz GA, Willett WC, Stampfer MJ, et al. Patterns of weight change and their relation to diet in a cohort of healthy women. Am J Clin Nutr 1990; 51: 1100-5.

42. van Dam RM, Stampfer M, Willett WC, et al. Dietary fat and meat intake in relation to risk of type 2 diabetes in men. Diabetes Care 2002; 25: 417-24.

43. Lawton CL, Delargy HJ, Brockman J, et al. The degree of saturation of fatty acids influences post-ingestive satiety. Br J Nutr 2000; 83: 473-82.

44. Flint A, Jørgensen BH, Raben A, et al. No differences in appetite and energy expenditure after intake of polyunsaturated, monounsaturated or trans-fatty acids. Obes Res 2003; in press.

45. Wolever TMS, Jenkins DJA, Vuksan V, et al. Beneficial effect of low-glycemic index diet in overweight NIDDM subjects. Diabetes Care 1992; 15: 562-4.

46. Wolever TMS, Mehling C. High-carbohydrate-lowglycaemic index dietary advice improves glucose disposition index in subjects with impaired glucose tolerance. Br J Nutr 2002; 87: 477-87.

47. Wolever TMS, Mehling C. Long-term effect of varying the source of dietary carbohydrate on postprandial plasma glucose, insulin, triacylglycerol, and free fatty acid concentrations in subjects with impaired glucose tolerance. Am J Clin Nutr 2003; 77: 612-21.

\section{Arne Astrup}

Department of Human Nutrition, The Royal Veterinary and Agricultural University, Rolighedsvej 30, DK-1958 Frederiksberg C, Denmark

E-mail: ast@kvl.dk 